\title{
A Simple Capacitor Voltage Balancing Method with a Fundamental Sorting Frequency for Modular Multilevel Converters
}

\author{
Hao Peng ${ }^{*}$, Ying Wang ${ }^{*}$, Kun Wang ${ }^{*}$, Yan Deng ${ }^{\dagger}$, Xiangning He*, and Rongxiang Zhao* \\ ${ }^{* \dagger}$ College of Electrical Engineering, Zhejiang University, Hangzhou, China
}

\begin{abstract}
A Fundamental Frequency Sorting Algorithm (FFSA) is proposed in this paper to balance the voltages of floating dc capacitors for Modular Multilevel Converters (MMCs). The main idea is to change the sequences of the CPS-PWM carriers according to the capacitor voltage increments during the previous fundamental period. Excessive frequent sorting is avoided and many calculating resources are saved for the controller. As a result, more sub-modules can be dealt with. Furthermore, it does not need to measure the arm currents. Therefore, the communication between the controllers can be simplified and the number of current sensors can be reduced. Moreover, the proposed balancing method guarantees that all of the switching frequencies of the sub-modules are equal to each other. This is quite beneficial for the thermal design of the sub-modules and the lifetime of the power switches. Simulation and experimental results acquired from a 9-level prototype verify the viability of the proposed balancing method.
\end{abstract}

Key words: Carrier Phase Shift PWM (CPS-PWM), Dual Sorting Mechanism, Fundamental Frequency Sorting Algorithm (FFSA), Modular Multilevel Converter (MMC)

\section{INTRODUCTION}

The Modular Multilevel Converter (MMC) has attracted a great deal of attention due to its outstanding performance in High Voltage Direct Current (HVDC) transmission and Flexible AC Transmission Systems (FACTS) during the last decade, especially after the Trans Bay Cable Project was successfully accomplished by Siemens [1]. When compared with diode or flying capacitor clamped multilevel converters, the MMC has many merits such as modular construction, redundancy, low switching frequency, low losses and low harmonics [1]-[3]. From recent publications, MMC-related studies are mainly focused on modulation strategies [3]-[8], modeling [9], [10], theoretical calculations [11], [12] and capacitor voltage balancing methods [4], [5], [13]-[17]. The capacitor voltage balancing method is critical for MMCs, as it is related to the safe operation of the whole system and has a significant impact on the output waveform.

Manuscript received Jun. 2, 2014; accepted Oct. 2, 2014

Recommended for publication by Associate Editor Rae-Young Kim.

${ }^{\dagger}$ Corresponding Author: dengyan@zju.edu.cn

Tel: +86-571-87952416, Fax: +86-571-87951797, Zhejiang University

*College of Electrical Engineering, Zhejiang University, China
Additional hardware circuits are used to balance the capacitors in [13]. The capacitors are clamped one by one by the diodes so that the capacitor voltage of the top sub-module and the bottom sub-module must be the highest or the lowest. Thus, all of the capacitor voltages are equal when the capacitors of the top and bottom sub-modules are balanced by an additional isolated circuit. This method simplifies the controllers and is easy to realize. However, it needs an extra circuit, especially since the isolated transformer between the top and bottom sub-modules undertakes a high insulation voltage which is equal to the voltage of the DC main bus.

An open-loop balancing method with a special modulation strategy that is executed at the fundamental switching frequency without measuring the capacitor voltage is presented in [14]. It rotates the switching angles of the sub-modules at the fundamental frequency to keep the capacitor voltage stable over a large number of fundamental periods. This method saves a lot of voltage sensors. However, it cannot balance the capacitors well when the load or the DC bus voltage changes.

A closed-loop control method which adds an extra signal to the modulation signal is presented in [7], [15]. With this method, the modulation and the individual voltage closed-loop 
control tasks are assigned to sub-module controllers, while the output voltage control, the power control and the averaging voltage control of the capacitors are done by the central controller. This distributed control structure is consistent with modular architecture. The parameters of the closed-loop controllers have to keep the system stable. Nevertheless, the difficulty of the control and the risk of instability increase with an increase of the sub-modules.

The sorting algorithm is a commonly used balancing method which can adapt to all of the modulations. Its core idea is to calculate how many sub-modules should be inserted into the circuit by the modulation strategy. Then the most suitable sub-modules are selected. This method is both simple and practical. However, there are frequent sorting issues with the capacitor voltage which are a burden to the controller, especially in the case of a large number of sub-modules. Moreover, the sub-modules are inserted and bypassed randomly so that the switching frequencies of the sub-modules are different from each other. This is harmful for the thermal design and the lifetime of the power switches due to uneven power losses and heat distribution. [4], [5], [16] reduce the calculation scale of each sorting to save calculation resources. The sorting frequency is reduced by controlling the high frequency circulating current to balance the capacitor voltages with CPS-PWM [17]. [18] adopts a counting unit to calculate the total number of switching commutations and it uses this information to keep the switching commutations evenly distributed. However, frequent sorting and uneven switching commutations cannot be solved at the same time with these methods.

In high power application, the switching frequencies of the power switches should be limited due to thermal limitations. In this paper, a CPS-PWM with fundamental carrier frequency is adopted for the Modular Multilevel Converter in order to obtain the fundamental switching frequency. At the same time, a Fundamental Frequency Sorting Algorithm (FFSA) with a dual sorting mechanism is proposed. It uses capacitor voltage increments during the previous fundamental period to judge which carrier of the CPS-PWM charges the most and the least. The first step is to sort the voltage increments of the capacitors in descending order and the present capacitor voltages in ascending order. Then, let the carrier which corresponds to the largest voltage increment drive the sub-module whose capacitor voltage is the lowest. After that, operate with the other carriers in turn. The arm current does not need to be measured. With this method, the sorting frequency and all of the switching frequencies of the power switches are equal to the fundamental frequency.

This paper is organized as follows. The structure and operation principle of the MMC are presented in Section II. A detailed analysis and operation process of the FFSA are introduced in Section III. Simulation results obtained from a 9-level inverter model are illustrated in Section IV to show the

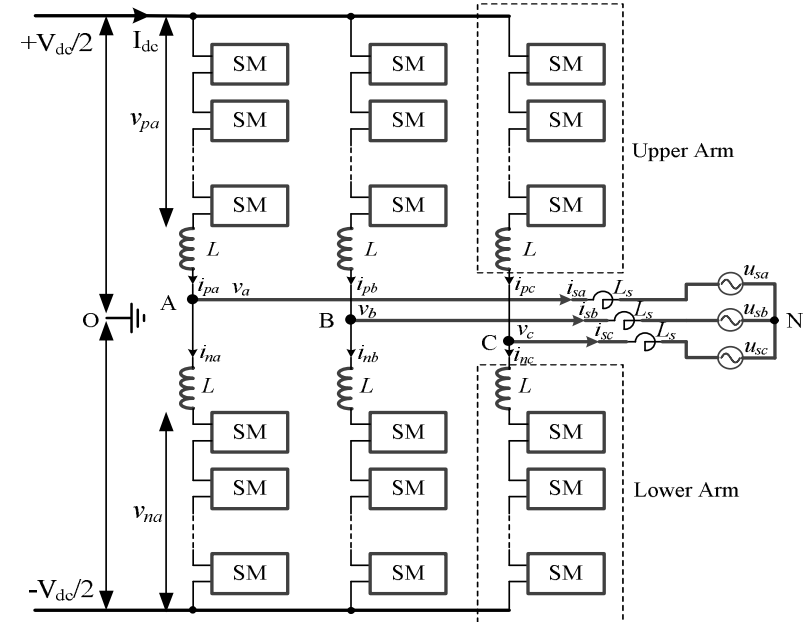

(a) MMC topology.

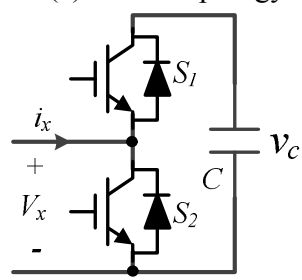

(b) Configuration of sub-module.

Fig. 1. Structure of MMC.

correctness of the FFSA. In Section V, experimental results are carried out to verify the proposed balancing method. The last section summarizes the conclusions.

\section{STRUCTURE AND OPERATION PRINCIPLES OF THE MMC}

\section{A. Structure of the $M M C$}

The typical structure of a three-phase MMC is shown in Fig. 1(a) and the configuration of a sub-module is presented in Fig. 1(b). Each phase consists of one upper arm and one lower arm, which are connected in series between two DC terminals. Each arm includes $\mathrm{N}$ identical sub-modules and one buffer inductor $L$. The sub-module is a simple bi-directional chopper cell composed of two IGBTs, $S_{1}$ and $S_{2}$, and one DC capacitor, $C$. When $S_{1}$ is on and $S_{2}$ is off, the SM is in the inserted state and the capacitor can be charged or discharged depending on the current direction. When $S_{1}$ is off and $S_{2}$ is on, the SM is in the bypassed state. During these two controlled states, the terminal voltage of the SM can be either zero or the capacitor voltage.

\section{B. Steady-State Analysis}

For one phase of the MMC, define half of the summation of the upper arm current and lower arm current as the circulating current $i_{c i r j}(j=a, b, c)$ :

$$
\left\{\begin{array}{l}
i_{c i r j}=\left(i_{p j}+i_{n j}\right) / 2 \\
i_{p j}=i_{c i r j}+i_{s j} / 2 \\
i_{n j}=i_{c i r j}-i_{s j} / 2
\end{array}\right.
$$


where $i_{p j}$ and $i_{n j}$ are the upper arm current and the lower arm current of phase $j(j=a, b, c)$, respectively, and $i_{s j}$ is the output current of phase $j(j=a, b, c)$.

The KVL equations of the upper arm and the lower arm can be listed as:

$$
\left\{\begin{array}{l}
v_{j}=\frac{1}{2} V_{d c}-v_{p j}-L \frac{d i_{p j}}{d t} \\
v_{j}=-\frac{1}{2} V_{d c}+v_{n j}+L \frac{d i_{n j}}{d t}
\end{array}\right.
$$

where $v_{p j}$ and $v_{n j}$ are the upper arm voltage and lower arm voltage of phase $j(j=a, b, c)$, respectively, $V_{d c}$ is the DC bus voltage and $v_{j}$ is the output voltage of phase $j(j=a, b, c)$.

From (1) and (2):

$$
\left\{\begin{array}{l}
v_{j}=\frac{1}{2}\left(v_{n j}-v_{p j}\right)-\frac{1}{2} L \frac{d i_{s j}}{d t} \\
L \frac{d i_{c i r j}}{d t}=\frac{1}{2} V_{d c}-\frac{1}{2}\left(v_{n j}+v_{p j}\right)
\end{array}\right.
$$

It can be inferred that the output voltage depends on the voltage difference between the upper arm and the lower arm. In addition, the circulating current is controlled by the voltage summation of the upper arm and the lower arm. The fundamental frequency of the output voltage and current at the ac side of the MMC can be described as:

$$
\left\{\begin{array}{l}
v_{j}=V_{s m} \sin \left(\omega t+\theta_{V}\right) \\
i_{s j}=I_{s m} \sin \left(\omega t+\theta_{I}\right)
\end{array}\right.
$$

where $\theta_{V}$ and $\theta_{I}$ are the initial phase angles of the output voltage and current, and $V_{s m}$ and $I_{s m}$ are the amplitude of the output voltage and current.

If the power losses are neglected, the active power of the ac side and the dc bus should be equal.

$$
3 V_{s m} I_{s m} \cos \theta / 2=V_{d c} \times I_{d c}
$$

where $I_{d c}$ is the DC input current and $\theta$ is the load impedance angle, $\theta=\theta_{V}-\theta_{I} . \theta$ is in the range of $[-\pi / 2, \pi / 2]$.

Then the following equation can be acquired:

$$
I_{d c}=\frac{3 V_{s m} I_{s m} \cos \theta}{2 V_{d c}}=\frac{3}{4} m_{V} I_{s m} \cos \theta
$$

where $m_{V}$ is the voltage modulation index, $m_{V}=V_{s m} /\left(V_{d c} / 2\right)$.

The arm voltage and current can be calculated as [19]:

$$
\begin{gathered}
\left\{\begin{array}{l}
v_{p j}=\frac{V_{d c}}{2}-v_{j}=\frac{V_{d c}}{2}\left(1-m_{V} \sin \left(\omega t+\theta_{V}\right)\right) \\
v_{n j}=\frac{V_{d c}}{2}-v_{j}=\frac{V_{d c}}{2}\left(1+m_{V} \sin \left(\omega t+\theta_{V}\right)\right)
\end{array}\right. \\
\left\{\begin{array}{l}
i_{p j}=\frac{I_{d c}}{3}+\frac{i_{s j}}{2}=\frac{I_{s m}}{2}\left[\frac{1}{2} m_{V} \cos \theta+\sin \left(\omega t+\theta_{I}\right)\right] \\
i_{n j}=\frac{I_{d c}}{3}-\frac{i_{s j}}{2}=\frac{I_{s m}}{2}\left[\frac{1}{2} m_{V} \cos \theta-\sin \left(\omega t+\theta_{I}\right)\right]
\end{array}\right.
\end{gathered}
$$

[11] and [20] calculate the arm current, analyze the fluctuation of the capacitor voltage in detail, and point out that the total energy of the arm can be balanced naturally in the steady state.

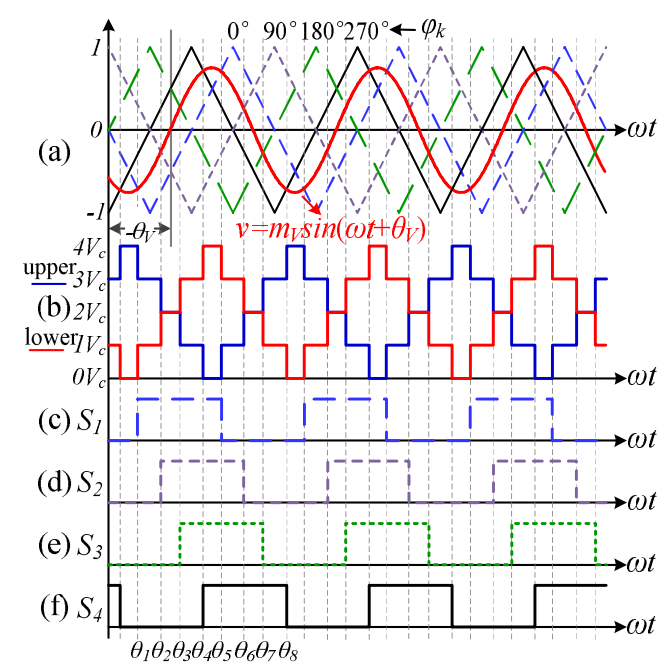

Fig. 2. CPS-PWM modulation process.

\section{DERIVATION OF THE FUNDAMENTAL FREQUENCY SORTING ALGORITHM}

\section{A. CPS-PWM with a Fundamental Carrier Frequency}

In high power applications, the power switches are always operated at a low switching frequency due to a power loss limitation. In this paper, CPS-PWM with a fundamental carrier frequency is adopted for a Modular Multilevel Converter, in order to limit the switching losses. The 5-level CPS-PWM modulation process with a fundamental carrier frequency is carried out as an example in Fig. 2. The initial phase angles of the $\mathrm{N}$ carriers $\varphi_{k}(k=1,2, \cdots, \mathrm{N})$ are $0,2 \pi / \mathrm{N}, \cdots,(\mathrm{k}-1) 2 \pi / \mathrm{N}, \cdots$, $(\mathrm{N}-1) 2 \pi / \mathrm{N}$. The stair waveforms of the upper arm voltage and the lower arm voltage are shown in Fig. 2(b). The drive signals $S_{k}(k=1,2, \cdots, \mathrm{N})$ for the lower arms are generated by the $\mathrm{N}$ carriers and the modulation signal, as shown in Fig. 2(c), (d), (e) and (f). The $\mathrm{N}$ drive signals for the upper arm are opposite to the $\mathrm{N}$ drive signals for the lower arm. The switching angles of the $k^{\text {th }}$ drive signal $S_{k}$ are $\theta_{k}$ and $\theta_{N+k}$. Considering the symmetry of the carriers and the modulation waveforms, the switching angles satisfy (9).

$$
\begin{cases}m_{V} \sin \left(\theta_{k}+\theta_{V}\right)=-\frac{2}{\pi}\left(\theta_{k}+2 q \pi-\varphi_{k}\right), & k=1,2, \cdots, N \\ \theta_{N+k}=\pi+\theta_{k}, & k=1,2, \cdots, N\end{cases}
$$

where $q$ is an integer, $q=-\infty, \cdots,-1,0,1, \cdots,+\infty$.

Unfortunately, the exact expression of $\theta_{k}$ is very complicated and hard to calculate. It depends on the modulation index $m_{V}$, the phase angle of the output voltage $\theta_{V}$ and the initial phase angles of the carriers $\varphi_{k}$. When the modulation signal is kept stable, $m_{V}$ and $\theta_{V}$ are constant so that only $\theta_{k}$ depends on $\varphi_{k}$. The Fourier series expansion of the $k^{\text {th }}$ drive signal $S_{k}$ can be expressed as:

$$
d_{k}=\frac{1}{2}+\frac{2}{\pi} \sum_{m=1,3,5, \cdots}^{\infty} \frac{1}{m} \sin m\left(\omega t-\theta_{k}\right), \quad k=1,2, \cdots, N
$$

As a special case, there are six crossing points between a few 


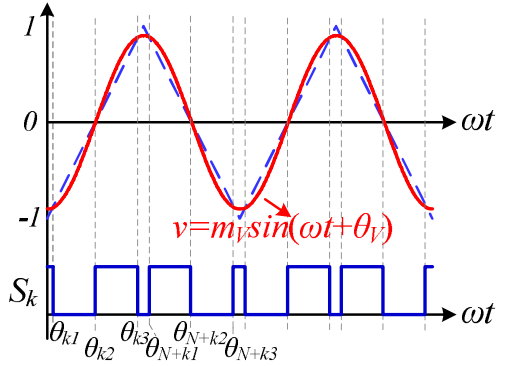

Fig. 3. Six crossing points for CPS-PWM under specific condition.

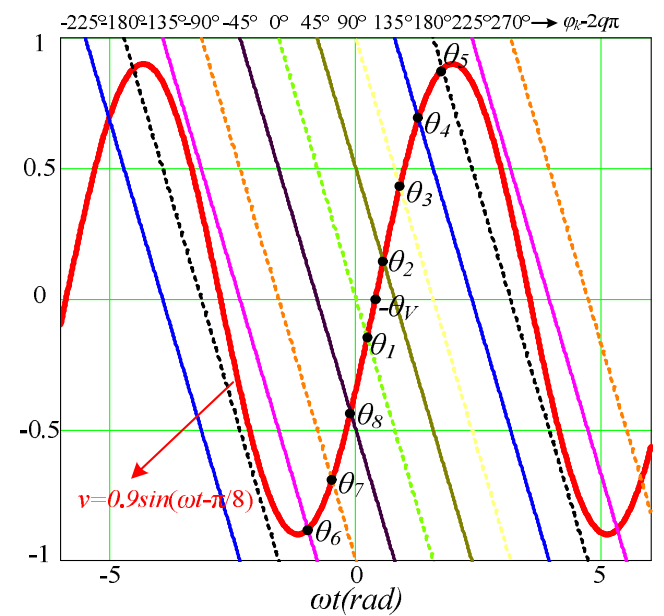

Fig. 4. Solving the switching angles with Mathcad.

carriers and the modulation waveforms when $m_{V}>2 / \pi$, as shown in Fig. 3. Suppose the switching angles are $\theta_{k 1}, \theta_{k 2}, \theta_{k 3}$, $\theta_{N+k 1}, \theta_{N+k 2}, \theta_{N+k 3}$. With the method mentioned above, the FFT expressions of the $k^{\text {th }}$ driving signals $S_{k}$ can be analyzed as:

$$
\begin{aligned}
d_{k}= & \frac{1}{2}+\frac{2}{\pi} \sum_{m=1,3,5, \ldots}^{\infty} \frac{1}{m}\left[-\sin m\left(\omega t-\theta_{k 1}\right)\right] \\
& +\frac{2}{\pi} \sum_{m=1,3,5, \ldots}^{\infty} \frac{1}{m}\left[\sin m\left(\omega t-\theta_{k 2}\right)\right] \\
& +\frac{2}{\pi} \sum_{m=1,3,5, \ldots}^{\infty} \frac{1}{m}\left[-\sin m\left(\omega t-\theta_{k 3}\right)\right] \\
= & \frac{1}{2}+\frac{2}{\pi} \sum_{m=1,3,5, \ldots}^{\infty} \frac{A_{m k}}{m} \sin m\left(\omega t-\theta_{k}\right)
\end{aligned}
$$

where $A_{m k}$ and $\theta_{k}$ relate to the three switching angles $\theta_{k 1}, \theta_{k 2}$ and $\theta_{k 3}$. Considering that the summation of $\theta_{k 1}$ and $\theta_{k 3}$ is approximately $2 \pi, A_{m k}$ is near to 1 and $\theta_{k}$ is approximately $\theta_{k 2}$.

(10) and (11) can be simplified to an approximate unified expression as:

$$
d_{k}=\frac{1}{2}+\frac{2}{\pi} \sum_{m=1,3,5, \cdots}^{\infty} \frac{1}{m} \sin m\left(\omega t-\theta_{k}\right), k=1,2,3, \cdots, N
$$

For carriers that have only two crossing points, $\theta_{k}$ is just the switching angle. For carriers that have six crossing points, $\theta_{k}$ relates to the three switching angles $\theta_{k 1}, \theta_{k 2}$ and $\theta_{k 3}$. $\theta_{k}$ is approximately $\theta_{k 2}$.

Fig. 4 presents a graph method to obtain the solution of $\theta_{k}$ for a 9-level MMC with Mathcad software, where the modulation index $m_{V}$ is 0.9 , the initial phase angle of the modulation signal $\theta_{V}$ is $-\pi / 8$ and the number of sub-modules in one arm $\mathrm{N}$ is 8 . It can be inferred that $\theta_{k}$ is nearly evenly distributed in the range $\left[-\theta_{V}-\pi / 2,-\theta_{V}+\pi / 2\right]$. If the equivalent switching angle $\beta_{k}$ is defined as (13), then $\beta_{k}$ is in the range $[-\pi / 2, \pi / 2]$.

$$
\beta_{k}=\theta_{k}+\theta_{V}, k=1,2,3, \cdots, N
$$

(12) can be derived as:

$$
d_{k}=\frac{1}{2}+\frac{2}{\pi} \sum_{m=1,3,5, \ldots}^{\infty} \frac{1}{m} \sin m\left(\omega t+\theta_{V}-\beta_{k}\right)
$$

\section{B. Capacitor Voltage Balance Analysis}

Under the CPS-PWM with a fundamental carrier frequency, the capacitor voltage increments of the lower arm sub-modules can be calculated by:

$$
\Delta v_{c k}=\frac{1}{C} \int_{0}^{t} d_{k} \times i_{n j} d t
$$

The capacitor voltage increment $\Delta v_{c k}$ contains a dc component, the fundamental frequency and its integer multiple components. Therefore, the capacitor voltage increment in a fundamental period can be calculated by:

$$
\Delta V_{c T k}=\frac{1}{C} \int_{0}^{T} d_{k} \times i_{n j} d t=\frac{I_{s m}}{\omega C}\left[\frac{\pi}{4} m_{V} \cos \theta-\cos \left(\theta-\beta_{k}\right)\right]
$$

The capacitor voltage increment is proportionate to the load current and it relates to the power factor of the load and the modulation index. Define $I_{s m} / \omega C$ as the normalized reference of the voltage increment so that the normalized voltage increment can be obtained.

$$
\Delta V_{c T k}{ }^{*}=\Delta V_{c T k} / \frac{I_{s m}}{\omega C}=\frac{\pi}{4} m_{V} \cos \theta-\cos \left(\theta-\beta_{k}\right)
$$

The relationship between the voltage increment during a fundamental period and the equivalent switching angle $\beta$ is indicated in Fig. 5. It can be seen that some carriers can charge the capacitors, while the others can discharge the capacitors in a fundamental period. Thus, the capacitors will diverge soon without a balancing method.

\section{FFSA based Balancing Method}

As described above, $\mathrm{N}$ drive signals can be generated by the CPS-PWM. However, the capacitor voltage of the $\mathrm{N}$ sub-modules will be unbalanced if the $\mathrm{N}$ sub-modules are driven with a fixed sequence. Since different carriers have a different impact on the capacitors, alternating the sequence of the carriers in a fundamental period can change the charging state of the sub-modules. The capacitor voltage increments in a fundamental period can be used as criterion to judge which carrier can charge the most and which can discharge the most. Then let the carrier charging the most drive the sub-module with the lowest capacitor voltage, and let the carrier charging the second most to drive the sub-module with the second lowest capacitor voltage. Then deal with the other carriers in 


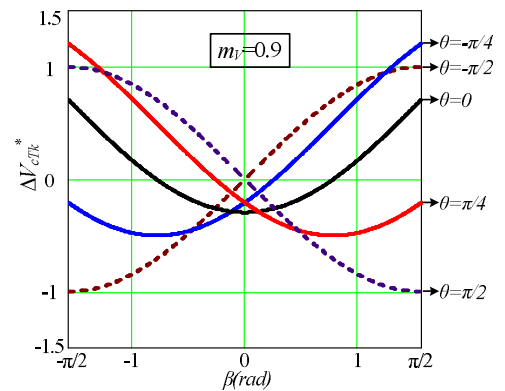

(a) Influence of the load impedance angle $\theta$.

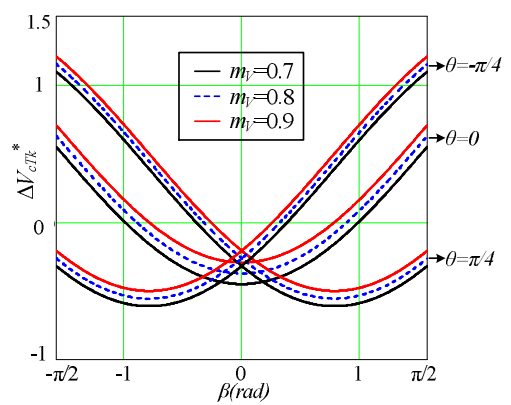

(b) Influence of the modulation index $m_{V}$.

Fig. 5. capacitor voltage increment vs equivalent switching angles.

the same way. In this way, the $\mathrm{N}$ drive signals can match the $\mathrm{N}$ sub-modules one by one with the best sequence.

The detailed operation process of a FFSA based on the dual sorting mechanism is shown in Fig. 6. Firstly, calculate the capacitor voltage increments during the previous fundamental period. Then sort the capacitor voltage increments in descending order and sort the present capacitor voltage in ascending order at the minimum point of the output voltage. The next step is to determine the carriers corresponding to the voltage increments and the sub-modules corresponding to the present capacitor voltage. At last, the re-matching relationship between the carriers and the sub-modules can be carried out and the driving sequence can be changed immediately. Note that the sorting and the driving sequence change can guarantee the fundamental sorting frequency and avoid the extra switching commutation because all of the sub-modules are bypassed at the minimum point of the output voltage with $\mathrm{N}+1$ levels.

The sorting frequency can be effectively reduced with the FFSA. However, the calculation resources consumed in one sorting process increase due to the dual sorting mechanism. This problem can be solved through the following three ways. Firstly, the calculation resources can be saved by dividing the whole sorting process into many asynchronous parts so that these parts can share some of the logic resources in the Field Programmable Gate Array (FPGA) controller. Even though the executing speed of the sorting process is reduced, this method has little influence on the performance of the FFSA due to its low sorting frequency. Secondly, the relationship curve of the

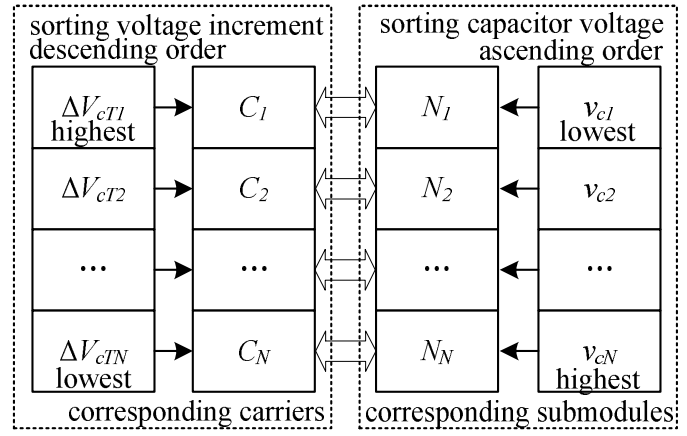

Fig. 6. FFSA with dual sorting mechanism.

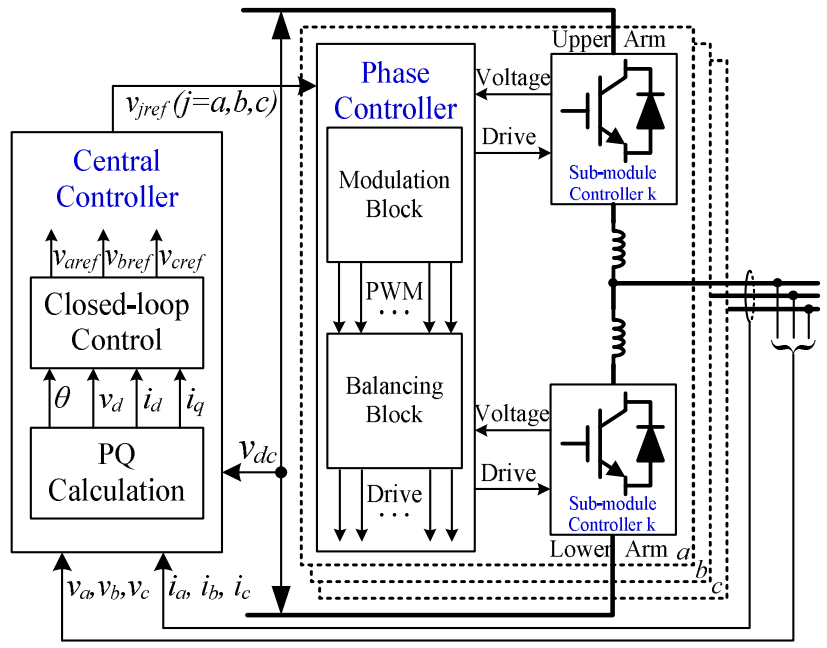

Fig. 7. Implementation of the proposed balancing method.

voltage increment and the switching angle has a minimum point, as shown in Fig. 5. The voltage increment enlarges with an increase in the absolute difference between the equivalent switching angle $\beta$ and the minimum point. With this method, only after the minimum point is determined by the sorting algorithm, the sorting result of the voltage increments can be carried out. Thus, the calculation resources can be greatly saved. Thirdly, it does not need to sort the present voltages completely. Only the largest and lowest voltages, which are beyond the specified range, should be determined. Let the carriers which can discharge the most drive the sub-modules with the highest voltage, let the carriers which can charge the most drive the sub-modules with the lowest voltage, and let the other carriers drive the remaining sub-modules. With these methods, the calculation resources can be reduced a lot.

The proposed balancing method is implemented with the control system shown in Fig. 7. The whole control system includes three-level controllers. The central controller is composed of a DSP and a FPGA, while each of the phase controllers is made up of a FPGA. The main task of the central controller is to calculate the active power and the reactive power and to implement the closed-loop control algorithm. The modulation process and the balancing algorithm are executed 


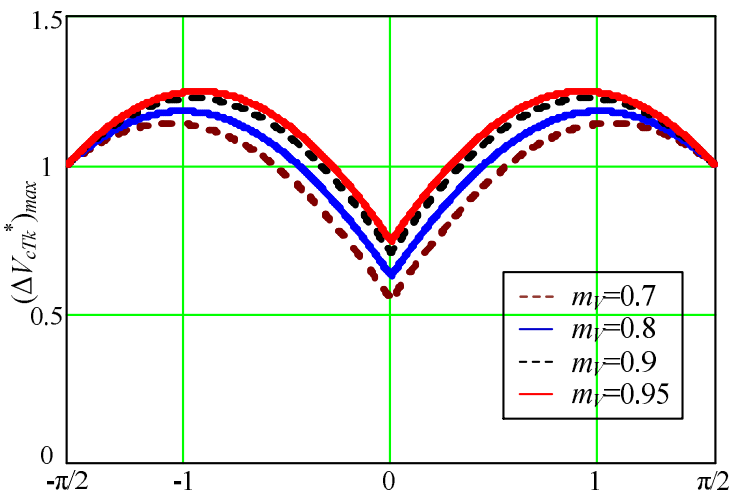

Fig. 8. The maximum ripple of DC bias with different load.

in the phase controller. The PWM signals generated by the modulation block should not drive the sub-modules directly. They are arranged in a proper order by the balancing block. The sub-module controller, which consists of a DSP, generates two driving signals with dead time according to the driving signals transmitted from the phase controller and it samples the capacitor voltage of the sub-module.

\section{Capacitor Voltage Ripple with the FFSA}

There are two factors that make the capacitor voltage fluctuate. One is the AC fluctuation whose ripple frequency is a multiple of the fundamental frequency. The other is the fluctuation of the DC bias. The rated sub-module capacitor voltage is $1 / \mathrm{N}$ of the DC bus voltage $V_{d c}$. However, the DC bias fluctuates with the FFSA. When $\beta_{k}$ is $\pi / 2$ or $-\pi / 2$, the DC bias in one fundamental period can obtain its maximum value.

$$
\left(\Delta V_{c T k}{ }^{*}\right)_{\max }=\frac{\pi}{4} m_{V} \cos \theta+\sin |\theta|
$$

Note that $\beta_{k}$ is the equivalent switching angle and $\beta_{k}$ is a discrete constant so that (18) is an appropriate expression. The curve of the maximum ripple of the DC bias is shown in Fig. 8. With a different load or a different modulation index, the maximum ripple varies. It can be seen that the maximum value of the maximum ripple of the capacitor voltage occurs when the load is a resistive-inductive or a resistive-capacitive load, not a pure resistor, inductor or capacitor. When the load impedance angle is (19), the maximum ripple of the DC bias obtains its maximum value (20). When the load is a pure resistive load, the maximum ripple of the DC bias acquires its minimum value $\pi m_{V} / 4$.

$$
\begin{gathered}
\theta= \pm \arctan \frac{4}{\pi m_{V}} \\
\left(\Delta V_{c T k}\right)_{\max }=\frac{I_{s m}}{\omega C}\left(1+\frac{\pi m_{V}}{4}\right) \frac{1}{\sqrt{1+\left(\frac{4}{\pi m_{V}}\right)^{2}}}
\end{gathered}
$$

(20) can be used as a criterion to choose the DC capacitance, in order to limit the ripple of the DC bias of the capacitor voltage.
TABLE I

SPECIFICATION OF THE SimULATION MODEL

\begin{tabular}{lc}
\hline \multicolumn{1}{c}{ Parameters } & Value \\
\hline DC bus voltage $\left(V_{d c}\right)$ & $6000 \mathrm{~V}$ \\
Modulation index $\left(m_{V}\right)$ & 0.9 \\
Output frequency $(f)$ & $50 \mathrm{~Hz}$ \\
Number of SMs in each arm $(N)$ & 8 \\
Arm inductance $(L)$ & $30 \mathrm{mH}$ \\
SM capacitance $(C)$ & $3 \mathrm{mF}$ \\
Load inductance $\left(L_{s}\right)$ & $15 \mathrm{mH}$ \\
Load resistance $(R)$ & $75 \Omega$ \\
Rated capacitor voltage $\left(V_{c}\right)$ & $750 \mathrm{~V}$ \\
Equivalent switching frequency $\left(N f_{s w}\right)$ & $400 \mathrm{~Hz}$ \\
\hline
\end{tabular}

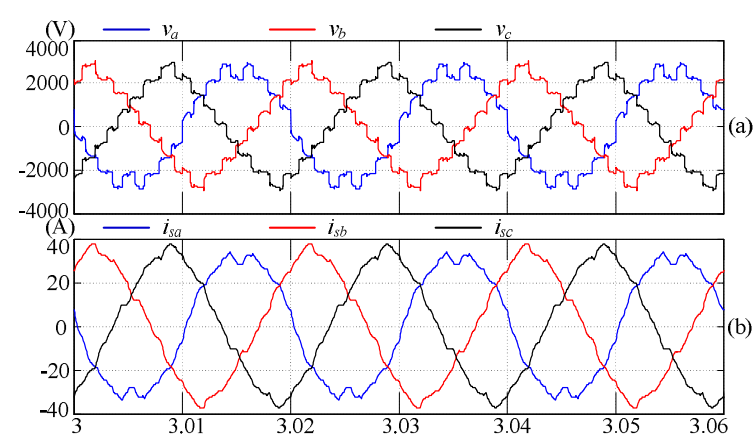

Fig. 9. Output voltage and current.

\section{SimUlation VerificATION}

In order to verify the effectiveness of the proposed balancing method, the 9-level three phase inverter shown in Fig. 1 is simulated in MATLAB. The detailed specifications of the whole system are listed in Table I.

The output voltage and current waveforms are shown in Fig. 9. The voltage of phase $a$ is different from phase $b$ and phase $c$ because of the six crossing points between a few carriers and the modulation signal. For the FFSA, the corresponding relationship between the carriers and the sub-modules changes every fundamental period, as shown in Fig. 10. The sorting flag is shown in Fig. 10(b), and the carriers corresponding to the first and fourth sub-module in the upper arm and lower arm of phase a are presented in Fig. 10 (c), (d), (e) and (f). The drive signals for the first, third, fifth and seventh sub-modules in the upper arm of phase a are indicated in Fig. 11 (a), (b), (c) and (d). The summation of all the drive signals for the upper arm of phase a is shown in Fig. 11(e). From these simulation results, the switching frequencies of all of the sub-modules are equal.

Fig. 12 demonstrates the perfect performance of the proposed balancing method, regardless of the steady state or dynamic state. At $0.7 \mathrm{~s}$, the startup of the simulation system is finished and all of the capacitors are charged to $750 \mathrm{~V}$. Then, the inverter is working in an open loop with no balancing algorithm. The capacitor voltages are divergent and start to converge when the FFSA is effective at $0.75 \mathrm{~s}$. The capacitor 


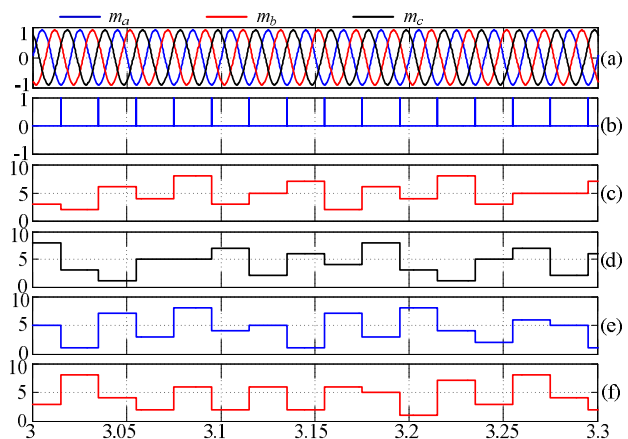

Fig. 10. Changing process of the drive sequence with FFSA.

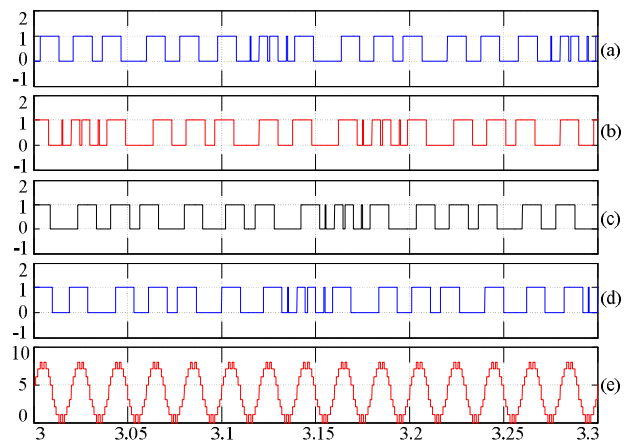

Fig. 11. Drive signals of the upper switch in each sub-module.

voltages are eventually stable at $750 \mathrm{~V}$. When the load is changed at $1.8 \mathrm{~s}$, the capacitor voltages can also be stable. The voltage ripples are a little bit larger under a heavy load. At $2.3 \mathrm{~s}$, the input voltage starts to decrease with a slope of $25000 \mathrm{~V} / \mathrm{s}$ until $5000 \mathrm{~V}$. With the FFSA, the capacitor voltages can be well balanced and stable at $625 \mathrm{~V}$. It can be inferred that the performance of the FFSA is quite good regardless of input voltage or load changes.

\section{EXPERIMENTAL RESULTS}

A down-scaled one-phase prototype is assembled to prove the proposed capacitor voltage balancing method. The configuration of the experimental system is presented in Fig. 13. The parameters of the prototype are indicated in Table II. The DC bus voltage is generated by an auto-transformer with a three phase diode rectifier.

Fig. 14 shows the experimental results of the CPS-PWM with a fundamental carrier frequency. The output voltage and arm voltages are all 9-level staircase waveforms. The switching frequencies of the fifth, the sixth sub-module of the upper arm and the fifth sub-module of the lower arm are equal to the fundamental frequency, as shown in Fig. 15. This proves that the switching frequencies between different sub-modules can be evenly distributed with the proposed balancing method.

The proposed FFSA-based capacitor voltage balancing method is verified with the open-loop output voltage control strategy. The DC bus voltage is $600 \mathrm{~V}$ and the modulation index remains at 0.9. Fig. 16 and Fig. 17 show the steady-state

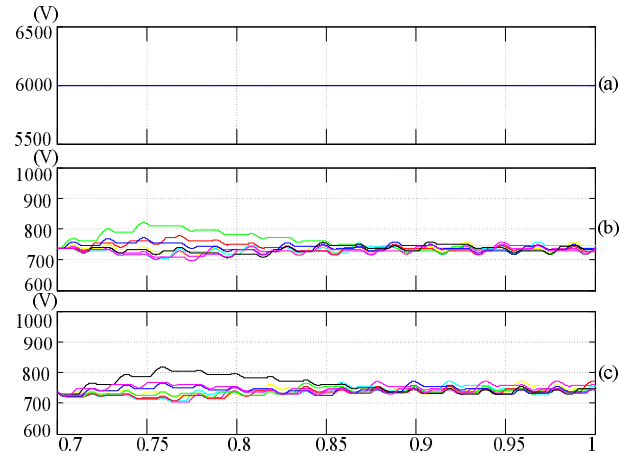

(a) Steady state result.

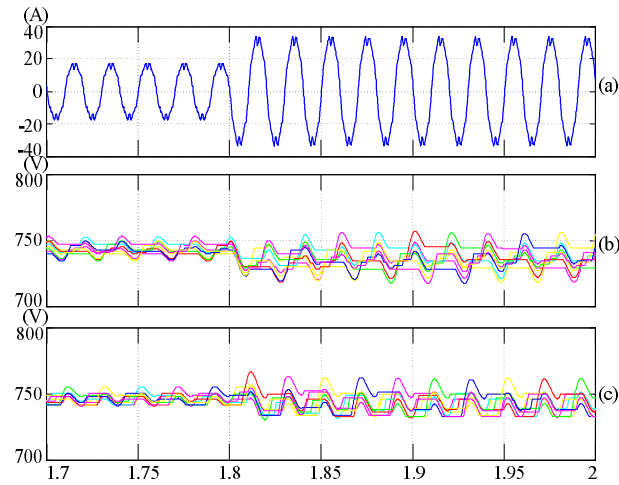

(b) Load increasing simulation.

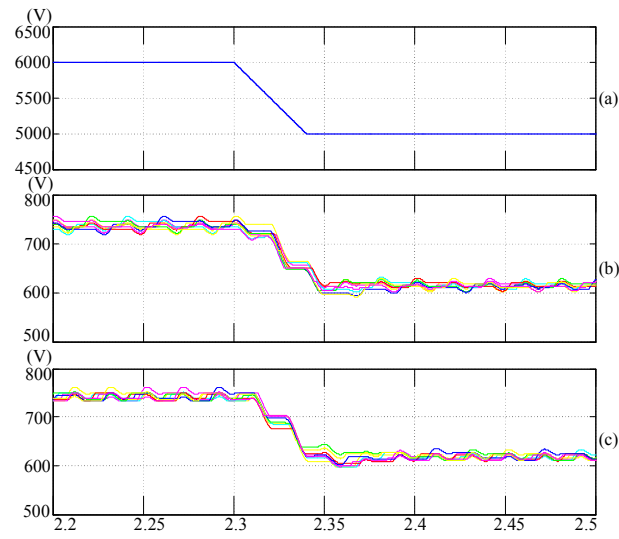

(c) Input voltage increasing simulation.

Fig. 12. Simulation results of the proposed balancing method.

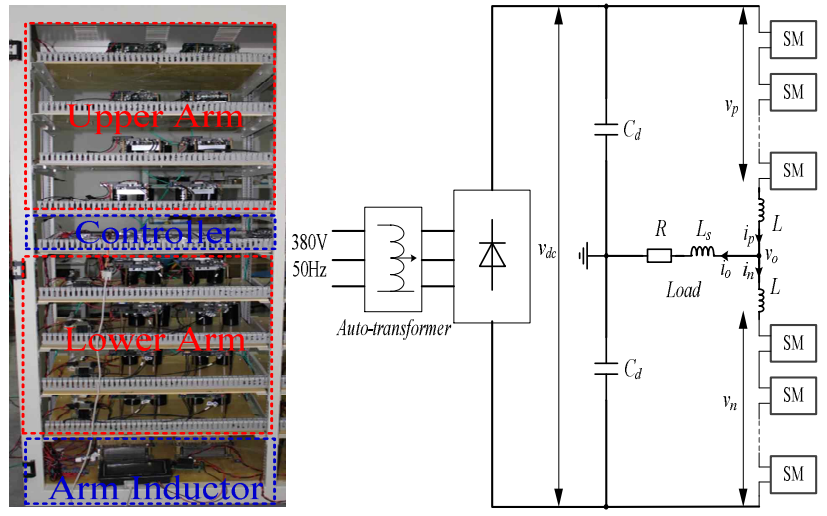

Fig. 13. Configuration of the experimental system. 
TABLE II

SPECIFICATION OF THE EXPERIMENTAL SySTEM

\begin{tabular}{lc}
\hline \multicolumn{1}{c}{ Parameters } & Value \\
\hline DC bus voltage $\left(V_{d c}\right)$ & $600 \mathrm{~V}$ \\
DC bus Capacitance $\left(C_{d}\right)$ & $3 \mathrm{mF}$ \\
Modulation index $\left(m_{V}\right)$ & 0.9 \\
Output frequency $(f)$ & $50 \mathrm{~Hz}$ \\
No of SMs in each arm $(N)$ & 8 \\
Arm inductance $(L)$ & $30 \mathrm{mH}$ \\
SM capacitance $(C)$ & $3 \mathrm{mF}$ \\
Load inductance $\left(L_{s}\right)$ & $15 \mathrm{mH}$ \\
Load resistance $(R)$ & $25 \Omega$ \\
Rated capacitor voltage $\left(V_{c}\right)$ & $75 \mathrm{~V}$ \\
Switching frequency $\left(f_{s w}\right)$ & $50 \mathrm{~Hz}$ \\
Equivalent switching frequency $\left(N f_{s w}\right)$ & $400 \mathrm{~Hz}$ \\
\hline
\end{tabular}

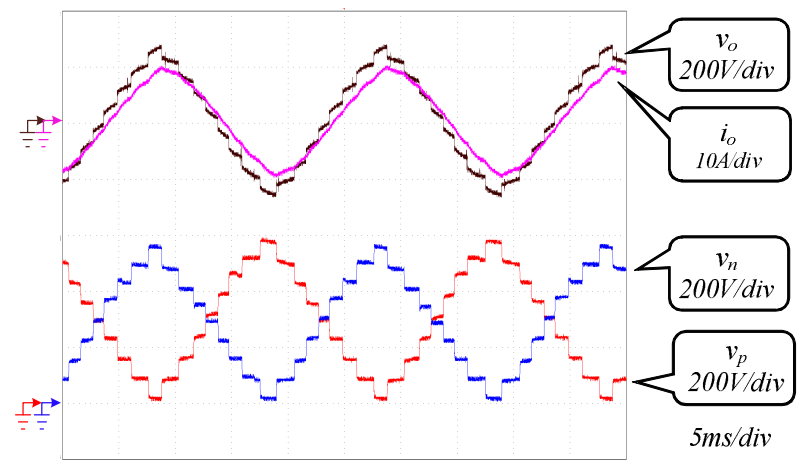

Fig. 14. Experimental results of CPS-PWM.

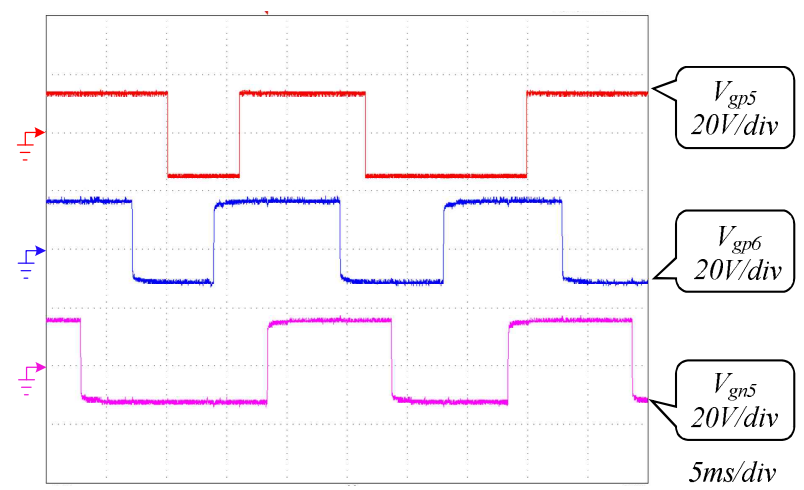

Fig. 15. Drive signals of the upper switch in each sub-module.

and dynamic results, respectively. For the steady state, the DC bias can be stable at $75 \mathrm{~V}$, and the ripple of the capacitor voltage is about $\pm 5 \mathrm{~V}$. For the dynamic performance, experimental results under the conditions where the load increases, the load decreases and the DC bus voltage increases are carried out. When the load resistance changes from $50 \Omega$ to $25 \Omega$ or from $25 \Omega$ to $50 \Omega$, the capacitor voltages can be stable at $75 \mathrm{~V}$. Only the ripples of the capacitor voltages increase a little under a heavy load. When the DC bus voltage increases from $300 \mathrm{~V}$ to $600 \mathrm{~V}$ by the auto-transformer, the DC bias of the capacitor voltages increases from $38 \mathrm{~V}$ to $75 \mathrm{~V}$. The capacitor voltages can be well balanced with the proposed FFSA-based capacitor voltage balancing method.

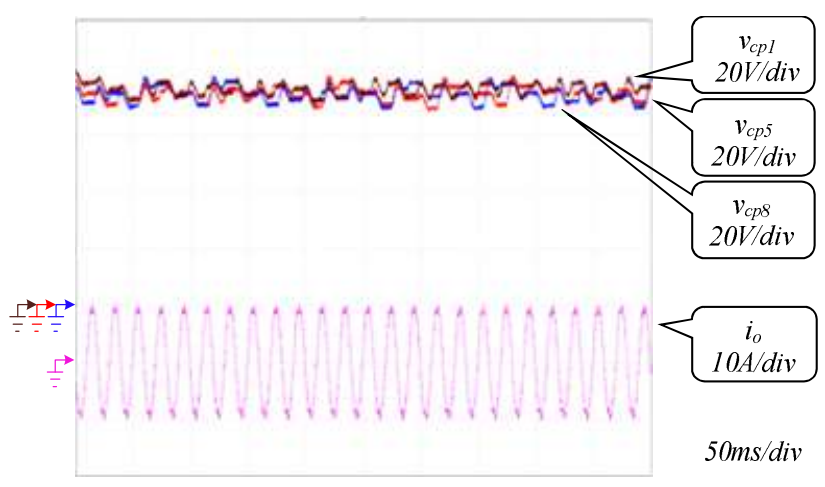

(a) Capacitor voltages of the upper sub-modules.

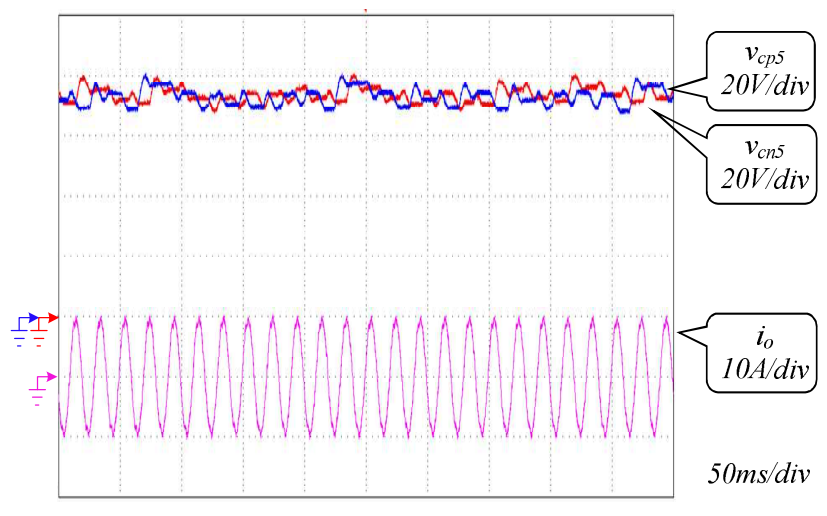

(b) Capacitor voltages of the upper arm and lower arm.

Fig. 16. Steady-state results of proposed balancing method.

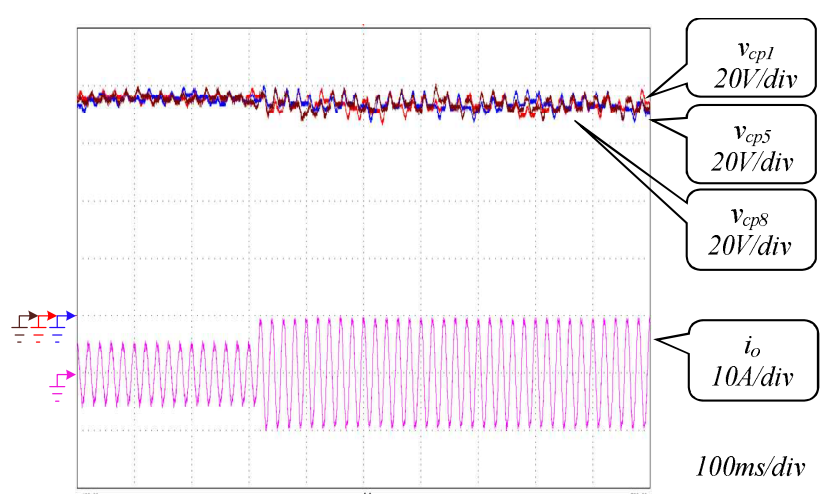

(a) Load increasing experiment.

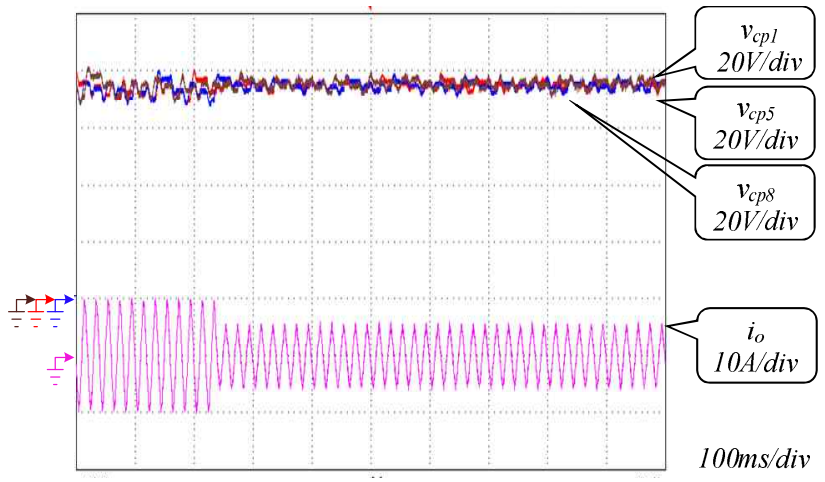

(b) Load decreasing experiment. 


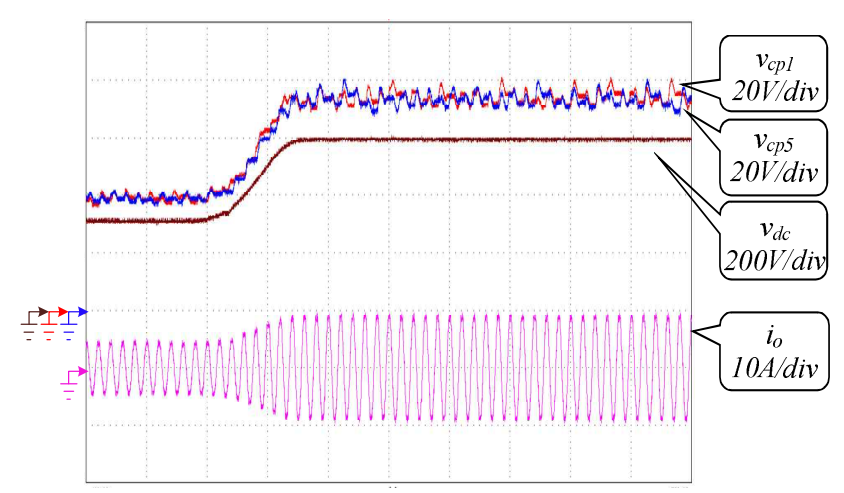

(c) Input voltage stepping up experiment.

Fig. 17. Dynamic results of proposed balancing method.

\section{CONCLUSIONS}

In this paper, a fundamental frequency sorting algorithm based on the dual sorting mechanism is proposed to balance the dc capacitor voltages of MMCs. Excessive frequent sorting can be avoided and a lot of calculation resources can be saved for the controllers. Thus, more sub-modules can be dealt with. This can also be adapted to the large-scale MMCs. Furthermore, the arm currents do not need to be measured so that the architecture of the control system can be simplified and the current sensors can be saved. Moreover, the switching frequencies of the switches are evenly distributed between the different sub-modules. As a result, the thermal design can be simplified. Simulation and experimental results verify the correctness of the proposed FFSA-based capacitor voltage balancing method.

\section{REFERENCES}

[1] K. Friedrich, "Modern HVDC PLUS application of VSC in Modular Multilevel Converter topology," in Industrial Electronics (ISIE), 2010 IEEE International Symposium on, pp. 3807-3810, 2010.

[2] A. Lesnicar and R. Marquardt, "An innovative modular multilevel converter topology suitable for a wide power range," in Power Tech Conference Proceedings, 2003 IEEE Bologna, Vol. 3, 2003.

[3] B. Gemmell, J. Dorn, D. Retzmann, and D. Soerangr, "Prospects of multilevel VSC technologies for power transmission," in Transmission and Distribution Conference and Exposition, 2008. T\&D. IEEE/PES, pp. $1-16,2008$.

[4] P. Meshram and V. Borghate, "A Simplified Nearest level control (NLC) Voltage Balancing Method for Modular Multilevel Converter (MMC)," IEEE Trans. Power Electron., Vol. 30, No. 1, pp. 450-462, Apr. 2014.

[5] J. Mei, K. Shen, B. Xiao, and L. Tolbert, "A new selective loop bias mapping phase disposition PWM with dynamic voltage balance capability for modular multilevel converter," IEEE Trans. Ind. Electron., Vol. 61, No. 2, pp. 798-807, Mar. 2013.

[6] S. Fan, K. Zhang, J. Xiong, and Y. Xue, "An improved control system for modular multilevel converters with new modulation strategy and voltage balancing control," IEEE
Trans. Power Electron., Vol. 30, No. 1, pp. 358-371, Feb. 2014.

[7] M. Hagiwara and H. Akagi, "Control and experiment of pulsewidth-modulated modular multilevel converters," IEEE Trans. Power Electron., Vol. 24, No. 7, pp. 1737-1746, Jul. 2009

[8] K. Shen, J. Wang, D. Zhao, M. Ban, Y. Ji, and X. Cai, "Investigation of capacitor voltage regulation in modular multilevel converters with staircase modulation," Journal of Power Electronics, Vol. 14, No. 2, pp. 282-291, Mar. 2014.

[9] A. Antonopoulos, L. Angquist, and H. Nee, "On dynamics and voltage control of the modular multilevel converter," in Power Electronics and Applications, 2009. EPE '09. 13th European Conference on, pp. 1-10, 2009.

[10] G. Casadei, R. Teodorescu, C. Vlad, and L. Zarri, "Analysis of dynamic behavior of Modular Multilevel Converters: Modeling and control," in System Theory, Control and Computing (ICSTCC), 2012 16th International Conference on, pp. 1-6, 2012.

[11] Y. S. Zhang, G. P. Adam, T. C. Lim, S. J. Finney, and B. W. Williams, "Mathematical analysis and experiment validation of modular multilevel converters," Journal of Power Electronics, Vol. 12, No. 1, pp. 33-39, Jan. 2012.

[12] W. Kui, L. Yongdong, Z. Zedong, and X. Lie, "Voltage balancing and fluctuation-suppression methods of floating capacitors in a new modular multilevel converter," IEEE Trans. Ind. Electron., Vol. 60, No. 5, pp. 1943-1954, May 2013.

[13] G. Congzhe, J. Xinjian, L. Yongdong, C. Zhe, and L. Jingyun, "A DC-link voltage self-balance method for a diode-clamped modular multilevel converter with minimum number of voltage sensors," IEEE Trans. Power Electron., Vol. 28, No. 5, pp. 2125-2139, May 2013.

[14] K. Ilves, A. Antonopoulos, S. Norrga, and H. P. Nee, "A new modulation method for the modular multilevel converter allowing fundamental switching frequency," IEEE Trans. Power Electron., Vol. 27, No. 8, pp. 3482-3494, Aug. 2012.

[15] M. Hagiwara, R. Maeda, and H. Akagi, "Control and analysis of the modular multilevel cascade converter based on double-star chopper-cells (MMCC-DSCC)," IEEE Trans. Power Electron., Vol. 26, No. 6, pp. 1649-1658, Jun. 2011.

[16] T. Qingrui, X. Zheng, and X. Lie, "Reduced switching-frequency modulation and circulating current suppression for modular multilevel converters," IEEE Trans. Power Del., Vol. 26, No. 3, pp. 2009-2017, Jul. 2011.

[17] F. Deng and Z. Chen, "A control method for voltage balancing in modular multilevel converters," IEEE Trans. Power Electron., Vol. 29, No. 1, pp. 66-76, Jan. 2014.

[18] G. T. Son, H.-J. Lee, T. S. Nam, Y.-H. Chung, U.-H. Lee, S.-T. Baek, K. Hur, and J.-W. Park, "Design and control of a modular multilevel HVDC converter with redundant power modules for noninterruptible energy transfer," IEEE Trans. Power Del., Vol. 27, No. 3, pp. 1611-1619, Jul. 2012.

[19] Y. Zhang, G. P. Adam, T. C. Lim, S. J. Finney, and B. W. Williams, "Mathematical analysis and experiment validation of modular multilevel converters," Journal of Power Electronics, Vol. 12, No. 1, pp. 33-39, Jan. 2012.

[20] X. Li, Q. Song, J. Li, and W. Liu, "Capacitor voltage balancing control based on CPS-PWM of modular multilevel converter," in Energy Conversion Congress and 


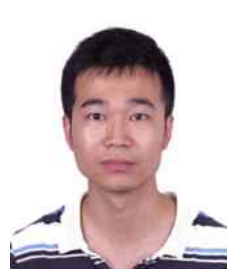

Hao Peng was born in Hubei Province, China, in 1989. He received his B.S. degree from the College of Electrical and Electronic Engineering, Huazhong University of Science and Technology, Wuhan, China, in 2010. He is currently working toward his Ph.D. degree in the College of Electrical Engineering, Zhejiang University, Hangzhou, China. His current research interests include high precision power amplifiers and modular multilevel converters.

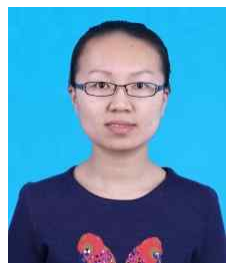

Ying Wang was born in China. She received her B.S. degree in Electrical Engineering and Automation from the University of Electronic Science and Technology of China, Chengdu, China, in 2012. Since September 2012, she has been a postgraduate student at Zhejiang University, Hangzhou, China, where she is currently working toward her M.S. degree in Power Electronics and Motor Drives. Her current research interests include Modular Multilevel Converters (MMCs) and HVDC Lights.

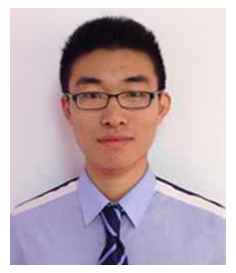

converters.
Kun Wang was born in China, in 1990. He received his B.S. degree from the University of Electronic Science and Technology of China, Chengdu, China, in 2013. He is currently working toward his M.S. degree in Electrical Engineering at Zhejiang University, Hangzhou, China. His current research interests include modular multilevel

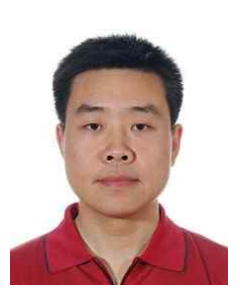

Yan Deng received his B.S. degree from the Department of Electrical Engineering, Zhejiang University, Hangzhou, China, in 1994, and his Ph.D. degree in Power Electronics and Electric Drives from the College of Electrical Engineering, Zhejiang University, in 2000. Since 2000, he has been a faculty member at Zhejiang University, where he is teaching and conducting research in the field of Power Electronics. He is currently an Associate Professor. His current research interests include the topologies and control methods for switch-mode power conversion.

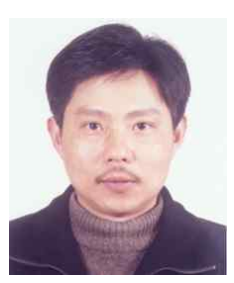

Xiangning He (M'95--SM'96--F'10) received his B.S. and M.S. degrees from the Nanjing University of Aeronautics and Astronautics, Nanjing, China, in 1982 and 1985, respectively. He received his Ph.D. degree from Zhejiang University, Hangzhou, China, in 1989. From 1985 to 1986, he was an Assistant Engineer at the 608 Institute of Aeronautical Industrial General Company, Zhuzhou, China. From 1989 to 1991, he was a Lecturer at Zhejiang University. In 1991, he obtained a Fellowship from the Royal Society of the U.K., and conducted research for two years in the Department of Computing and Electrical Engineering, Heriot-Watt University, Edinburgh, Scotland, U.K., as a Post-Doctoral Research Fellow. In 1994, he joined Zhejiang University as an Associate Professor. Since 1996, he has been a Full Professor in the College of Electrical Engineering, Zhejiang University. He was the Director of the Power Electronics Research Institute and the Head of the Department of Applied Electronics. He is currently the Vice Dean of the College of Electrical Engineering, Zhejiang University. His current research interests include power electronics and their industrial applications. He is the author or co-author of more than 200 papers and one book "Theory and Applications of Multi-level Converters." He currently holds 12 patents. Dr. He received a 1989 Excellent Ph.D. Graduate Award, a 1995 Elite Prize Excellence Award, a 1996 Outstanding Young Staff Member Award and a 2006 Excellent Staff Award from Zhejiang University for his teaching and research contributions. He received five Scientific and Technological Progress Awards from the Zhejiang Provincial Government and the State Educational Ministry of China in 1998, 2002 and $2009 \mathrm{He}$ has also received five Excellent Paper Awards. $\mathrm{He}$ is a Fellow of the Institution of Engineering and Technology (formerly IEE), Stevenage, England, U.K.

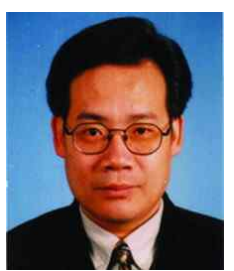

Rongxiang Zhao received his B.S., M.S. and $\mathrm{Ph} . \mathrm{D}$. degrees in Electrical Engineering from Zhejiang University, Hangzhou, China, in 1984 , 1987, and 1991, respectively. He is currently a Professor in the Department of Electrical Engineering, Zhejiang University, where he is also the Director of the National Engineering Research Center for Applied Power Electronics of China, the Industrial Technology Research Institute of Zhejiang University and the Innovation Center of Zhejiang University \& Fuji. His current research interests include micro-grids, renewable resources and their generation, motors and motor control, energy storage and its applications, and power converter systems. 